\title{
A Two-stage Hybrid Algorithm for Optic Camouflage Performance Assessment Based on DEA and ANN
}

\author{
Ling Li, Mengqi Cong, Heng Liu* \\ National Key Laboratory on Environmental Electromagnetic Effects and Electro-Optic Engineering \\ College of Field Engineering, PLA University of Science and Technology \\ Nanjing, China \\ liuhenglgdx@163.com
}

\begin{abstract}
This paper assessed optic camouflage performance by a two-stage hybrid model combined DEA and ANN. The assessment indexes of optic camouflage performance were firstly constructed. Then we proposed the hybrid algorithm by the following two stages: (1) Conventional CCR model was improved by super-efficiency issue with non-Archimedean infinitesimal; (2) ANN was combined with super-efficiency DEA to form a hybrid model. The objectivity of teacher value in ANN which is defined as the relative efficiencies of the optic camouflage performance has a bearing on convergence speed and learning precision of network. DEA is applied to calculate teacher values, which requires no assumption on the appearance of the frontier surface as well as makes no hypothesis concerning the internal operations of a decision making unit. Meanwhile, the super-efficiency scores will help to distinguish the efficient decision making units as well. A simulation test shows that the convergence speed of the new model is increased by $\mathbf{5 7 . 6 9 \%}$, and the learning error is improved by $98.47 \%$. The improved hybrid model has higher convergence speed and better learning precision than traditional one to assess optic camouflage performance.
\end{abstract}

Keywords-optic camuflage performance; assessment; superefficiency DEA; artificial neural networks; teacher value

\section{INTRODUCTION}

Assessment of camouflage performance has become an interesting topic of many scholars in recent years due to its vital role in camouflage technology. Optic camouflage performance plays an important role in the multi-band assessment. As we know, the assessment procedure is a multiple criteria decision making issue essentially, showing solicitude for suitable index set and effective evaluating measures.

Data envelopment analysis (DEA), originated by Charnes, Cooper, and Rhodes, is a non-parametric approach for assessing the relative efficiency of homogeneous decision making units (DMUs), which use similar inputs to produce similar outputs [1]. DEA evaluates the efficiency of each DMU relative to an estimated production possibility frontier formed by all DMUs. The advantage of DEA approach is that it requires no assumption on the appearance of the frontier surface as well as it makes no hypothesis concerning the internal operations of a DMU. In DEA model, the best DMUs are called DEA efficient and the efficiency score of a DMU is denoted by a unity, leading to unsatisfactory differentiation of all the DMUs. In order to solve this problem, sufficient ranking DEA efficient DMUs has become an popular topic of many scholars and a number of models (called superefficiency models) were put forward to rank DEA efficient DMU. Among these models, one can mention Andersen and Petersen's [2] super-efficiency model and the slack-based measure (SBM) introduced by Tone [3]. In recent years, the super-efficiency model for SBM has been developed by many researchers for the purpose of ranking SBM efficient DMUs [4] [5] [6]. A.A. Noura, et al. [7] developed the ranking method according to the weights and the desirability of inputs and outputs. In parallel, a number of researchers have studied the problem of super-efficiency model's infeasibility (see, e.g., [8] [9] [10]).

Artificial neural network (ANN) is a nonlinear selfadapting system, which can often be utilized to deal with complex input-output relationship. It provides a new way for feature extraction and classification [11] [12]. Backpropagation (BP) neural networks is the most extensively applied and can offer better solutions for multiple criteria decision making problem [13]. When BP neural networks are trained, some problems must be taken into consideration. Firstly, selection of the learning rate for nonlinear networks is a challenging issue. Secondly, settling in a local minimum may be good or bad depending on how close the local minimum is to the global minimum and how accurate an error is required. Thirdly, the network is sensitive to the number of neurons in its hidden layers. What's more, the objectivity of the teacher values of BP neural networks is significant in the evaluation procedure of multiple criteria.

In this paper, we suggested a two-stage hybrid algorithm, combining super-efficiency DEA and BP neural networks. It can be a good approach to approximate numerous nonparametric and nonlinear problems. Applying the hybrid model, we can assess the optic camouflage performance sufficiently as well as predict the efficiency scores of new DMUs. The rest of the paper is organized as follows. Section 2 proposes the assessment indices of optic camouflage 
performance. Section 3 suggests a hybrid two-stage model which combines super-efficiency DEA and ANN. Section 4 gives a numerical test and the results analysis.

\section{ASSESSMENT INDEX SYSTEM FOR OPTIC CAMOUFLAGE PERFORMANCE}

There are many elements that affect the performance of optic camouflage performance. We take index system into consideration from the follow principles: (1) Comprehensiveness and simplicity must be considered simultaneously to cover the most important influence indexes of optic camouflage performance. (2) Irrelevance should be thought over between every two indexes of input and output. (3) Availability of index data can be another crucial factor we must take into account.

Viewing DEA structure, input and output index have their normal requirements: input is inversely proportional to the DEA efficiency; output is proportional to the DEA efficiency. Thus, we classify the indexes into two categories of cost and benefit index, which can be described as input and output respectively. According to practical application of optic camouflage performance, we extract seven representative indexes to set up index system. We can classify them by two categories: we deal with brightness contrast $\left(x_{1}\right)$, exposure size $\left(x_{2}\right)$ and visibility distance $\left(x_{3}\right)$ as inputs, and handle speckle reflectivity $\left(y_{1}\right)$, speckle size $\left(y_{2}\right)$, speckle color $\mathrm{X}$ value $\left(y_{3}\right)$ and speckle color Y value $\left(y_{4}\right)$ as outputs. The index system can be illustrated as Fig.1.

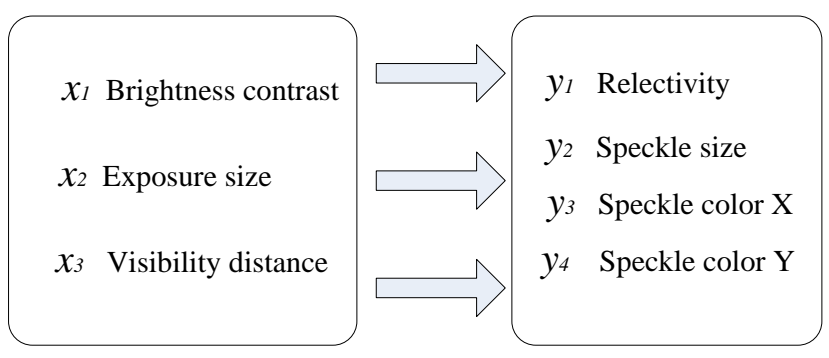

Inputs Indices

Outputs Indices

Figure 1. Assessment index system for optic camouflage performance

\section{Two-StAGE Hybrid Algorithm OF DEA-ANN}

\section{A. Improved Super-efficiency DEA Model}

DEA is a widely used mathematical programming approach for comparing the multiple inputs and outputs of a set of homogenous DMUs by measuring their relative efficiency. Suppose that there are $n$ DMUs to be measured where $j$ th DMU is defined as $D M U_{j}, j=1,2, \ldots, n$, consuming $m$ inputs, $x_{i j}(i=$ $1,2, \ldots, m)$, producing $s$ outputs, $y_{r j}(r=1,2, \ldots, s)$. The CCR model for evaluating the technical input-efficiency of $j_{0}$ th DMU $\left(D M U_{0}\right)$ under constant returns to scale (CRS) is represented as Eq. 1.

$$
\begin{array}{ll}
\max & q_{0}=\sum_{r=1}^{s} \mu_{r} y_{r 0} \\
\text { s.t. } & \sum_{i=1}^{m} \omega_{i} x_{i 0}=1 \\
& \sum_{i=1}^{m} \omega_{i} x_{i j}-\sum_{r=1}^{s} \mu_{r} y_{r j} \geq 0 \\
& j=1,2, \cdots n \\
& \omega_{i}, \mu_{r} \geq 0, \quad \forall i, r
\end{array}
$$

The dual model for assessing the input-orientated efficiency of $D M U_{0}$ under CRS with non-Archimedean infinitesimal is formulated as Eq. 2. Where $\hat{e}=(1,1, \ldots$, $1)^{T} \in E^{m}$ and $e=(1,1, \ldots, 1)^{T} \in E^{s}$ are unit vectors, and $\varepsilon$ $(\varepsilon>0)$ is a non-Archimedean infinitesimal. $S^{+}$and $S^{-}$are slacks, which reflect non-radial improvement between one DMU and its optimal condition. Slack based measure can provide us more management information about improvement. We construct the model under CRS, offering us overall efficiency, which includes technical efficiency under variable returns to scale (VRS) and scale effect.

$$
\begin{array}{cl}
\min & {\left[\theta-\varepsilon\left(\hat{e}^{T} S^{-}+e^{T} S^{+}\right)\right]} \\
\text {s.t. } & \sum_{j=1}^{n} X_{j} \lambda_{j}+S^{-}=\theta X_{0} \\
& \sum_{j=1}^{n} Y_{j} \lambda_{j}-S^{+}=Y_{0} \\
\lambda_{j} \geq 0, \quad j=1,2, \cdots, n \\
S^{-} \geq 0, S^{+} \geq 0
\end{array}
$$

The efficiencies of efficient DMUs in Eq. 2 are equal to unity simultaneously, which leads to unsatisfactory discrimination. Anderson and Petersen have suggested the super-efficiency model to arrive at a ranking of all efficient DMUs, which can be applied to differentiate efficient DMUs in DEA. Conventional DEA can be extended into SE-DEA, which is proposed as Eq. 3. Where, $\alpha$ is super-efficiency of SE-DEA.

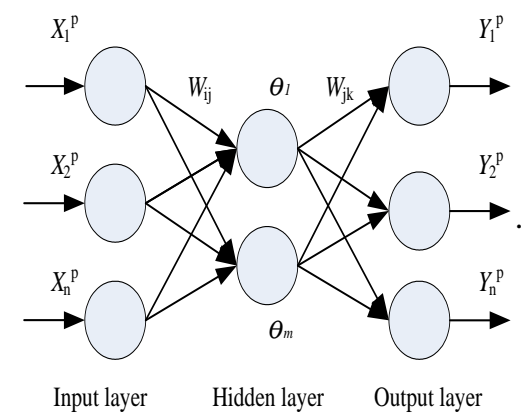

\section{B. Improved ANN Model}

ANN is constituted of processing elements (nodes or neurons) and connections. The nodes are interconnected layerwise of interconnection among themselves. Each node in the successive layer receives the inner product of synaptic weights, 
with the outputs of the nodes in the previous layer. Two types of learning networks are, respectively, supervised and unsupervised. In this research, a supervised learning network is more suitable for the multiple criteria decision making issue. For a supervised learning network, a set of training input vectors with a corresponding set of target vectors is trained to adjust the weights of ANN. In this paper, the target vector is the super-efficiency score of each DMU. Thus, it is a unit vector. BP neural network is the most popular of supervised ANN. With back-propagation the related input data are repeatedly presented to the neural networks. The output of the neural network is compared to the desired output and an error is calculated in each procedure. We can illustrate the structure of BP neural networks by Fig .2 as following.

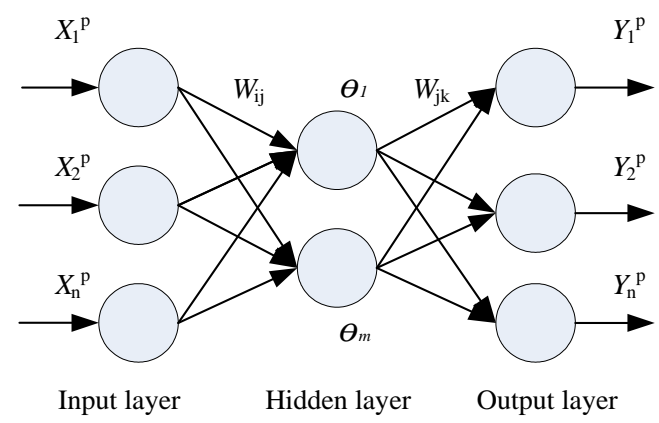

Figure 2. Structure of BP neural networks

BP network is composed of three layers: input, hidden and output layer. The number of input layer's and output layer's nerve cells depend on the dimensions of stylebook formed by data processor. The hidden layer's nerve cells are rectified by experiences. We define the error of BP neural networks as Eq. 4.

$$
E(x)=\frac{1}{2} \sum_{s=1}^{s} \sum_{i=1}^{l}\left(D_{i}{ }^{s}-D_{i}{ }^{s}\right)^{2} .
$$

Where, $D_{i}{ }^{S}$ and $D_{i}{ }^{S}$ is respectively the expectation output and actual output of $i$ th DMU in sth stylebook.

We define $f$ to be the transfer function of networks, which is formulated by Sigmoid function as Eq. 5 .

$$
f(x)=\frac{1}{1+e^{-x}} .
$$

In the procedure of optic camouflage performance assessment, the teacher value is provided by experts' evaluation or other ways. There are several methods of assessment of multiple criteria such as one-nine degree and experts mark, analytical hierarchy process [14], fuzzy comprehensive evaluation [15] method and so on. However, they are sometimes too "subjective". In order to provide a more "objective" assessment of optic camouflage performance, we introduce the approach of super-efficiency DEA model. It is a better way to organize and analyze data since it allows efficiency to change over time and requires no prior assumption on the specification of the best practice frontier.

\section{Two-stage Hybrid Algorithm}

Combine the super-efficiency DEA and ANN, we can integrate them into a two-stage hybrid model called BPSEDEA model.

Stage 1: Step 1. Differentiate the indices of optic camouflage performance assessment as inputs and outputs. Run super-efficiency DEA model, the optimum value of each DMU is obtained which is defined as super-efficiency score.

Stage 2: Step 2. Viewing the inputs and outputs data as the inputs of the ANN, the super-efficiency scores are treated as the teacher values of the networks. Train BP networks, we can get the training values of each DMU.

Step 3. Analyze the training results of DEA-ANN model by comparing the training values with the super-efficiency scores of pure super-efficiency DEA model. If the whole error $E(x)<\varepsilon$ (where $\varepsilon$ is a constant value), then stop training the networks.

Step 4. Apply the trained BP neural networks to forecast the other DMUs, which will give the trained values. They are the forecasting super-efficiency scores too.

Step 5. Rank the DMUs by forecasting scores. We can distinguish the performance of all the DMUs by ordering them sufficiently.

\section{NUMERICAL EAMPLE}

Now we test a numerical example using BP-SEDEA algorithm to illustrate its application procedure in the evaluation of optic camouflage performance. There are 15 DMUs with 3 inputs and 4 outputs.

We firstly applied CCR model and SEDEA model to analyze the data set respectively. The CCR efficiency scores and super-efficiency scores are viewed as the teacher values of BP-CCR and BP-SEDEA model respectively. We trained BPCCR and BP-SEDEA, and set the networks structure as 7-12-1. The maximum epochs are set to be 2000 , and the learning rate is defined as 0.6. The goal error of the BP-CCR networks is 0.1, and that of BP-SEDEA networks is set to be 0.01 .

$\mathrm{DMU}_{1}$ to $\mathrm{DMU}_{12}$ are applied to train the networks, and the last 3 DMUs are utilized to forecast the scores. The original data and the results are listed in Tab. I, from which, we can calculate the whole errors of the two networks. Where, the error of BP-CCR is 0.085, and the error of BP-SEDEA is 0.0013 , which is reduced by $98.47 \%$. The BP-SEDEA has a much better error than BP-CCR. The comparison figures are illustrated as Fig .3 and Fig .4. From Fig .3, we know that the $\mathrm{DMU}_{2}, \mathrm{DMU}_{5}, \mathrm{DMU}_{8}, \mathrm{DMU}_{10}, \mathrm{DMU}_{12}$ and $\mathrm{DMU}_{15}$ are all CCR efficient. The CCR scores are 1, which are hard to discriminate. The line function is $y=x$, the dots will be more precise if they are closer to the line. The CCR scores are not far from the BP-CCR training scores. 
TABLE I. DATA AND RESULTS ANALYSIS

\begin{tabular}{|c|c|c|c|c|c|c|c|c|c|c|c|}
\hline \multirow{2}{*}{$\mathbf{D M U}_{\mathbf{i}}$} & \multicolumn{3}{|c|}{ Inputs } & \multicolumn{4}{|c|}{ Outputs } & \multirow{2}{*}{$\begin{array}{l}\text { CCR } \\
\text { score }\end{array}$} & \multirow{2}{*}{$\begin{array}{c}\text { BP-CCR } \\
\text { score }\end{array}$} & \multirow{2}{*}{$\begin{array}{c}\text { SEDEA } \\
\text { score }\end{array}$} & \multirow{2}{*}{$\begin{array}{l}\text { BP-SEDEA } \\
\text { score }\end{array}$} \\
\hline & $x_{1}$ & $x_{2}$ & $x_{3}$ & $y_{1}$ & $y_{2}$ & $y_{3}$ & $y_{4}$ & & & & \\
\hline $\mathrm{DMU}_{1}$ & 1.125 & 48 & 82.5 & 0.66 & 9.75 & 16 & 15.8 & 0.744 & 0.732 & 0.744 & 0.743 \\
\hline $\mathrm{DMU}_{2}$ & 1.05 & 32 & 82.5 & 0.76 & 10.65 & 19.2 & 16 & 1 & 0.893 & 1.018 & 1.013 \\
\hline $\mathrm{DMU}_{3}$ & 1.2 & 41.6 & 102 & 0.62 & 10.35 & 16 & 15.4 & 0.641 & 0.632 & 0.641 & 0.639 \\
\hline $\mathrm{DMU}_{4}$ & 0.9 & 44.8 & 97.5 & 0.72 & 11.7 & 17.4 & 18 & 0.925 & 0.930 & 0.925 & 0.928 \\
\hline $\mathrm{DMU}_{5}$ & 0.975 & 35.2 & 69 & 0.76 & 11.55 & 17.8 & 16.4 & 1 & 0.886 & 1.115 & 1.092 \\
\hline $\mathrm{DMU}_{6}$ & 1.02 & 46.4 & 66 & 0.58 & 10.35 & 16 & 15 & 0.891 & 0.881 & 0.891 & 0.889 \\
\hline $\mathrm{DMU}_{7}$ & 1.05 & 48 & 135 & 0.66 & 12.9 & 16.4 & 14.4 & 0.741 & 0.749 & 0.741 & 0.745 \\
\hline $\mathrm{DMU}_{8}$ & 0.675 & 24 & 57 & 0.56 & 9 & 19.8 & 22 & 1 & 0.792 & 1.141 & 1.116 \\
\hline $\mathrm{DMU}_{9}$ & 0.75 & 27.2 & 61.5 & 0.5 & 10.2 & 19.2 & 20.6 & 0.954 & 0.948 & 0.954 & 0.953 \\
\hline $\mathrm{DMU}_{10}$ & 0.6 & 22.4 & 60 & 0.5 & 11.25 & 18.6 & 23 & 1 & 0.821 & 1.357 & 1.340 \\
\hline $\mathrm{DMU}_{11}$ & 0.84 & 44 & 84 & 0.6 & 10.8 & 16.2 & 19 & 0.838 & 0.841 & 0.838 & 0.836 \\
\hline $\mathrm{DMU}_{12}$ & 0.63 & 40 & 78 & 0.48 & 12.3 & 18 & 21.6 & 1 & 0.786 & 1.041 & 1.012 \\
\hline $\mathrm{DMU}_{13}$ & 0.99 & 37.6 & 102 & 0.78 & 9.9 & 15.4 & 17.8 & 0.936 & 0.922 & 0.936 & 0.930 \\
\hline $\mathrm{DMU}_{14}$ & 1.08 & 45.6 & 76.5 & 0.76 & 13.35 & 17.2 & 13.8 & 0.996 & 0.980 & 0.996 & 0.990 \\
\hline $\mathrm{DMU}_{15}$ & 0.9 & 42.4 & 94.5 & 0.8 & 9.45 & 15 & 16 & 1 & 0.844 & 1.067 & 1.053 \\
\hline
\end{tabular}

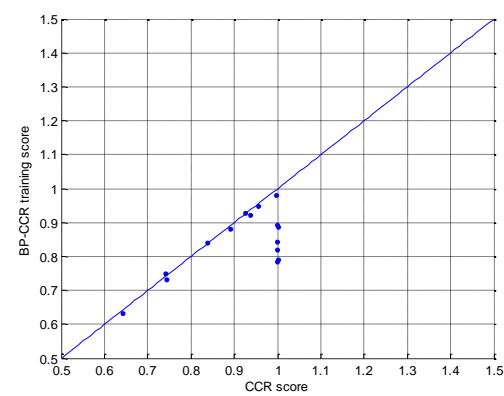

Figure 3. BP-CCR results comparison

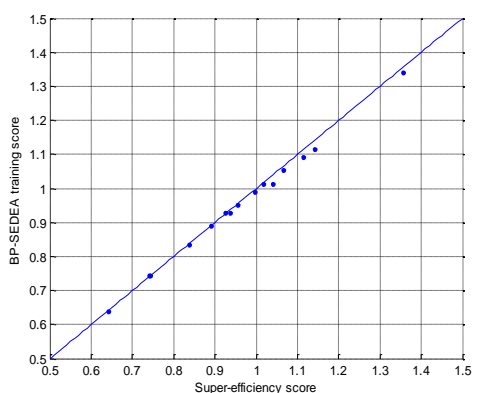

Figure 4. BP-SEDEA results comparison

However, SEDEA has better discernment ability. $\mathrm{DMU}_{2}$, $\mathrm{DMU}_{5}, \quad \mathrm{DMU}_{8}, \quad \mathrm{DMU}_{10}, \quad \mathrm{DMU}_{12}$ and $\mathrm{DMU}_{15}$ can be distinguished well with their super-efficiency scores, which are larger than a unity. We can also conclude that BP-SEDEA has better training precision. The dots in Fig .4 illustrated by superefficiency scores of $x$ axis and BP-SEDEA training scores of $y$ axis are closer to the line $y=x$.
Fig .5 and Fig .6 describe the training error constringency curve. From them, we can definitely conclude that BP-CCR networks constringency epochs is 26; that of BP-SEDEA networks is 11 . The convergence speed of the new model is increased by $57.69 \%$, showing a better constringency speed.

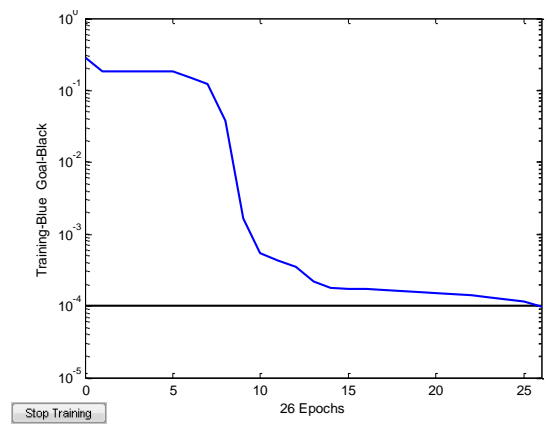

Figure 5. BP-CCR constringency curve

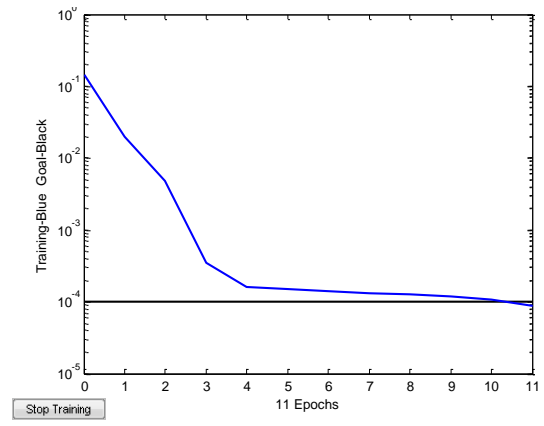

Figure 6. BP-SEDEA constringency curve

For SEDEA model, the ranking order of the efficient DMUs is obtained: $\mathrm{DMU}_{10} \succ \mathrm{DMU}_{8} \succ \mathrm{DMU}_{5} \succ \mathrm{DMU}_{15} \succ$ $\mathrm{DMU}_{12} \succ \mathrm{DMU}_{2}$. Where the symbol " $\succ$ " means "is superior 
to". But through the operation of BP-SEDEA, the rank changes: $\mathrm{DMU}_{10} \succ \mathrm{DMU}_{8} \succ \mathrm{DMU}_{5} \succ \mathrm{DMU}_{15} \succ \mathrm{DMU}_{2} \succ \mathrm{DMU}_{12}$. The order of $\mathrm{DMU}_{2}$ and $\mathrm{DMU}_{12}$ reverses.

\section{ACKNOWLEDGMENT}

This work is supported by Foundation of National Key Laboratory on Environmental Electromagnetic Effects and Electro-Optic Engineering (Grand No. FD2015008).

\section{REFERENCES}

[1] A. Charnes, W.W. Cooper, and E. Rhodes, "Measuring the efficiency of decision making units," European Journal of Operational Research, 2 (1978), pp. 429-444.

[2] P. Andersen, and N.C. Petersen, "A procedure for ranking efficient units in data envelopment analysis," Management Science, 39, 10(1993), pp. 1261-1264.

[3] K. Tone, "A slacks-based measure of efficiency in data envelopment analysis," European Journal of Operational Research, 130, 3(2001), pp. 498-509.

[4] Hsin-Hsiung Fang, Hsuan-Shih Lee, Shiuh-Nan Hwang, and Cheng-Chi Chung, "A slacks-based measure of super-efficiency in data envelopment analysis:An alternative approach," Omega-International Journal of Management Science, 41, 4(2013), pp. 731-734.

[5] Hsiao Bo, Chern Ching-Chin, Chiu Yung-Ho, and Chiu Ching-Ren, "Using fuzzy super-efficiency slack-based measure data envelopment analysis to evaluate Taiwan's commercial bank efficiency," Expert Systems with Applications, 38, 8(2011), pp. 9147-9156.

[6] Chiu Yung-Ho, Chen Yu-Chuan, and Bai Xue-Jie, "Efficiency and risk in Taiwan banking: SBM super-DEA estimation," Applied Economics, 43, 5(2011), pp. 587-602.
[7] A.A. Noura, F. Hosseinzadeh Lotfi, G.R. Jahanshahloo, and S. Fanati Rashidi, "Super-efficiency in DEA by effectiveness of each unit in society," Applied Mathematics Letters, 24, 5(2011), pp. 623-626.

[8] Esmaeilzadeh A., and Hadi-Vencheh A, "A super-efficiency model for measuring aggregative efficiency of multi-period production systems," Measurement, 46, 10(2013), pp. 3988-3993.

[9] Amirteimoori Alireza, and Kordrostami Sohrab, “A distance-based measure of super efficiency in data envelopment analysis: an application to gas companies," Journal of Global Optimization, 54, 1(2012), pp. 117-128.

[10] Chen Jin-Xiao, Deng Mingrong, and Gingras Sylvain, "A modified super-efficiency measure based on simultaneous input-output projection in data envelopment analysis," Computers \& Operations Research, 38, 2(2011), pp. 496-504.

[11] Dayhoff, J. E., Neural network architecture, New York: Van Nostrand Reinhold, 1990.

[12] Ghiassi M., Lio, David, and Moon Brian, "Pre-production forecasting of movie revenues with a dynamic artificial neural network," Expert Systems with Applications, 42, 6(2015), pp. 3176-3193.

[13] Huang Dai-Zheng, GongRen-Xi, and Gong Shu, "Prediction of Wind Power by Chaos and BP Artificial Neural Networks Approach Based on Genetic Algorithm," Journal of Electrical Engineering \& Technology, 10, 1(2015), pp. 41-46.

[14] C. H. Chou, G. S. Liang, and H. C. Chang, "A fuzzy AHP approach based on the concept of possibility extent," Quality \& Quantity,47, 1(2013), pp. 1-14.

[15] W. W. Chu, Y. G. Li, C. Q. Liu, W. P. Mou, and L. M. Tang, “A manufacturing resource allocation method with knowledge-based fuzzy comprehensive evaluation for aircraft structural parts," International Journal for Production Research, 52, 11(2014), pp. 3239-3258. 